\section{Nuclear receptor modulators: Catching information by machine learning}

Cecile Valsecchi,, ${ }^{\text {Francesca Grisoni, }}{ }^{2}$ Viviana Consonni, ${ }^{1}$ Davide Ballabio, ${ }^{1}$ Roberto Todeschini1

1Milano Chemometrics and QSAR Research Group, Department of Earth and Environmental Sciences, University of Milano Bicocca, Milan, Italy; 2Department of Chemistry and Applied Biosciences, ETH Zurich, Zurich, Switzerland

\begin{abstract}
Nuclear receptors (NRs) are involved in fundamental human health processes and are a relevant target for toxicological risk assessment. To help prioritize chemicals that can mimic natural hormones and be endocrine disruptors, computational models can be a useful tool.1,2 In this work we i) created an exhaustive collection of NR modulators and ii) applied machine learning methods to fill the data-gap and prioritize NRs modulators by building predictive models.
\end{abstract}

\section{Introduction}

The human nuclear receptor superfamily is composed by 48 key transcription factors, which constitute $16 \%$ of drug targets. ${ }^{3}$ Although the NRs ligand binding domain is highly conserved, NRs have been shown to be promiscuous targets, as demonstrated by the endocrine interference [4]. In this framework, in-silico modelling based on machine learning can be used to fill the data-gap in order to reduce animal testing and protect human health. Thus, we collected bioactivity data from different sources and then applied machine learning methods, with a particular focus on the simultaneous prediction of bioactivities for 8 NRs.

\section{Materials and Methods}

We created a publicly available dataset (NURA-NUclear Receptor Activity dataset $)^{5}$ containing annotations for binding, agonism and antagonism activity for 15,206 molecules and 8 selected NRs taken from 4 public databases (i.e., Tox21, ChEMBL, NR-DBIND and BindingDB). Each bioactivity type for a given receptor was considered as a task (e.g., binding activity for androgen receptor), obtaining a total of 30 tasks. The structural information of each molecule was encoded in a binary vector, i.e., as extended connectivity fingerprints (ECFPs). ${ }^{6}$ We applied two multi-task neural networks (FFNL1 and FFNL3), which allow to simultaneously model the 30 tasks. To assist the validation of in-silico models, molecules were randomly split into training set (11,970 molecules, 80\%) and test set (2,993 molecules, $20 \%)$.

\section{Results}

NURA dataset is a useful tool to bridge the gap between toxicology-and medicinalchemistry-related databases, as it is enriched in terms of number of molecules, structural diversity and covered atomic scaffolds compared to the single sources as shown in Figure 1 and Figure 2.

NURA dataset is intended to support decision-making in pharmacology and toxicology, as well as to contribute to data-driven applications. It can be downloaded at the following DOI: https://doi.org/10.5281/zenodo.3991561.

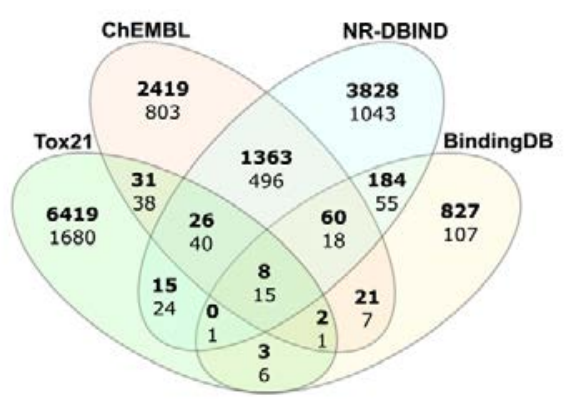

Figure 1. Venn diagram of the NURA data collected from Tox21, ChEMBL, NR-DBIND and Binding-DB and pruned. The numbers of shared and not shared molecules (in bold) and scaffolds are reported.

Correspondence: Cecile Valsecchi, Milano Chemometrics and QSAR Research Group, Department of Earth and Environmental Sciences, University of Milano Bicocca, P.za della Scienza 1, 20126 Milan, Italy.

E-mail: c.valsecchi18@campus.unimib.it

Key words: Nuclear Receptors; multi-task learning; machine learning; neural networks.

Disclosures: Authors have nothing to disclose.

Conference presentation: This paper was presented at the Third Centro 3R Annual Meeting - L'era delle 3R: modelli in silico, in vitro e in vivo per promuovere la ricerca traslazionale 30 September - 1 October 2021, Evento online organizzato dal Politecnico di Torino.

Received for publication: 9 July 2021

Accepted for publication: 7 September 2021.

This work is licensed under a Creative Commons Attribution NonCommercial 4.0 License (CC BY-NC 4.0).

(C) Copyright: the Author(s), 2021

Licensee PAGEPress, Italy

Biomedical Science and Engineering 2021; 4(s1):198 doi:10.4081/bse.2021.198

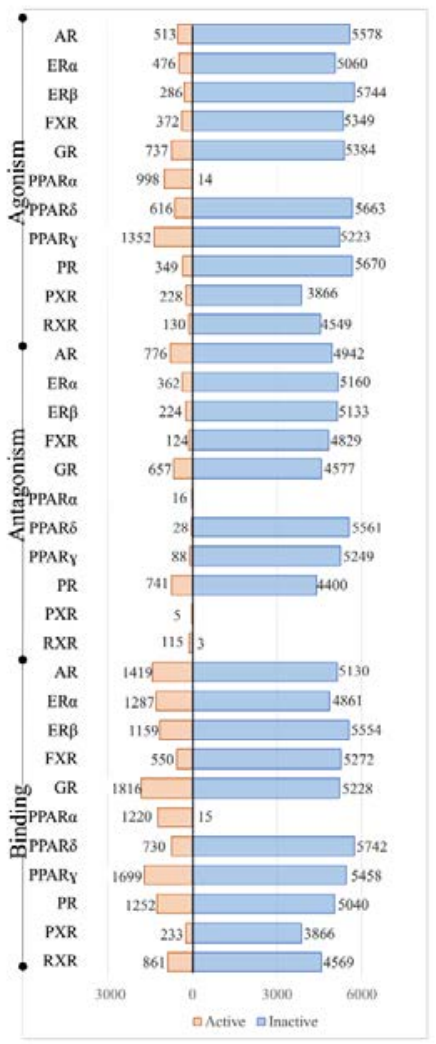

Figure 2. Distribution of molecules in the NURA dataset, divided into active (orange bar, activity lower than $10,000 \mathrm{nM}$ ), and inactive (blue bar, activity larger than $100,000 \mathrm{nM})$. 
Table 1. Global classification measures expressed as the percentage of correctly classified active $\left(\mathrm{Sn}_{\mathrm{T}}\right)$, inactive compounds $\left(\mathrm{Sp}_{\mathrm{T}}\right)$ and their average $\left(\mathrm{NER}_{\mathrm{T}}\right)$ achieved on the test set. ${ }^{7}$

\begin{tabular}{lccc} 
Model & NER & $S_{p T}$ & $S n_{T}$ \\
FFNL1 & 95.3 & 95.3 & 95.4 \\
FFNL3 & 95.2 & 95.4 & 95.1 \\
\hline
\end{tabular}

As shown in Table 1, all the in-silico approaches achieved good classification performances on test chemicals with a $95 \%$ of correctly classified compounds.

\section{Discussion and Conclusions}

The increased coverage of chemical and bioactivity space offered by NURA dataset result in a broader applicability domain and improved robustness of the developed computational models, which can help reducing animal testing by filling the data-gap and prioritizing chemicals. Furthermore, multi- task neural networks might offer other advantages, such as i) the possibility of leveraging information on related tasks, ii) modelling less represented tasks and iii) identifying ligands with selective or promiscuous activity.

\section{References}

1. Mansouri K, Abdelaziz A, Rybacka A, et al. CERAPP: Collaborative Estrogen Receptor Activity Prediction Project. Environ Health Perspect 2016;124:1023-33.
2. Mansouri K, Kleinstreuer N, Abdelaziz AM, et al. CoMPARA: Collaborative Modeling Project for Androgen Receptor Activity. Environ Health Perspect 2020;128:027002.

3. Santos R, Ursu O, Gaulton A, et al. A comprehensive map of molecular drug targets. Nat Rev Drug Discov 2017;16:19-34.

4. Germain P, Staels B, Dacquet C, et al. Overview of nomenclature of nuclear receptors. Pharmacol Rev 2006;58:685704.

5. Valsecchi C, Grisoni F, Motta S, et al. NURA: A curated dataset of nuclear receptor modulators. Toxicol Appl Pharmacol 2020;407:115244.

6. Rogers D, Hahn M. Extended-connectivity fingerprints. J Chem Inf Model. 2010 May 24;50(5):742-54.

7. Valsecchi C, Collarile M, Grisoni F, et al. Predicting molecular activity on nuclear receptors by multitask neural networks. J Chemometr 2020, https://doi.org/10.1002/cem.3325 